\title{
The Long and the Short of It: Relational Memory Impairments in Amnesia, Even at Short Lags
}

\author{
Deborah E. Hannula, ${ }^{1}$ Daniel Tranel, ${ }^{2}$ and Neal J. Cohen ${ }^{1,3}$ \\ ${ }^{1}$ University of Illinois, Urbana-Champaign, Illinois 61801, ${ }^{2}$ University of Iowa, Iowa City, Iowa 52242, and ${ }^{3}$ Washington University, St. Louis, Missouri \\ 63130
}

Classic studies of amnesia led to characterizations of hippocampal function emphasizing involvement in long-term memory rather than short-term (or working) memory. In two experiments, we show that when memory for relations among co-occurring items is tested, hippocampal amnesia results in a deficit even at very short lags. Hence, we find evidence for hippocampal involvement in relational memory, even at short lags normally considered the province of working memory.

Key words: memory; amnesia; hippocampus; relational memory; long-term memory; short-term memory; human

\section{Introduction}

Fifty years of neuropsychological research, conducted after severe memory loss was documented in patient H.M. (Scoville and Milner, 1957), has established that the hippocampus and surrounding medial temporal lobe (MTL) structures are critical for establishing new long-term declarative memories (Cohen and Squire, 1980; Cohen, 1984). Historically, amnesia has been described as a selective long-term memory impairment, leaving short-term or working memory intact (Scoville and Milner, 1957; Baddeley and Warrington, 1970). This view is supported by patient data showing strong delay- and lag-sensitivity of the deficit (Holdstock et al., 1995, 1999, 2000a,b; Buffalo et al., 1998) and normal performance on verbal and nonverbal tests of short-term memory (Wickelgren, 1968; Baddeley and Warrington, 1970; Warrington and Baddeley, 1974; Cave and Squire, 1992).

Despite evidence supporting the idea that amnesia affects long-term memory selectively, there are some reports of shortdelay memory impairments, even when just 5 or 6 s separate the cue from the corresponding probe stimulus (Holdstock et al., 1995; Buffalo et al., 1998). However, these impairments were observed in patients of mixed etiology, so questions remain about whether or not the hippocampus contributes to performance on tasks considered to be the province of short-term or working memory.

\footnotetext{
Received Dec. 7, 2005; revised June 28, 2006; accepted June 29, 2006.

This work was supported by grants from the National Institute of Mental Health and the National Science Foundation (N.J.C.) and by a National Institute of Neurological Disorders and Stroke Program Project Grant (D.T.). We would like to extend our gratitude to the patients, their families, and the comparison participants for taking the time to participate in these experiments. In addition, we are very grateful to Tressie Dust, Anjali lyengar, and Paul Menocc who helped create the materials that were used in experiment 1, to Ruth Henson and Kathy Jones at the University of lowa for the time and effort that they spent scheduling our visits with the patients, to Dr. Melissa Duff for her patient liaison efforts, and to Jake Gibbons, who provided technical assistance and guidance. Finally, our thanks to Ken Norman and to one anonymous reviewer for their helpful suggestions during several rounds of the review process.

Correspondence should be addressed to Deborah Hannula, 1544 Newton Court, Center for Neuroscience, University of California, Davis, CA 95616. E-mail: dehannula@ucdavis.edu.

DOI:10.1523/JNEUROSCI.5222-05.2006

Copyright $\odot 2006$ Society for Neuroscience $\quad$ 0270-6474/06/268352-08\$15.00/0
}

Research on the nature of declarative memory over the past two decades has led to a focus on relational memory. Our group in particular has suggested that the hippocampus is required for the formation and flexible use of representations of all manner of relations among items in scenes or events (Cohen and Eichenbaum, 1993; Ryan et al., 2000; Eichenbaum and Cohen, 2001).

This recent emphasis on relational memory has been largely limited to long-term memory tasks, but studies revealing lagsensitivity of the deficit in amnesia, and disproportionate impairment on long-term memory tasks, have been largely limited to memory for items, and tend to be conducted with patients of mixed etiology (Holdstock et al., 1995, 1999; Buffalo et al., 1998). This leaves open whether hippocampal function and hippocampal amnesia are truly selective for long-term memory, or whether the hippocampus might also contribute to performance at short delays, in the absence of intervening items between study and test, when relational memory is required. A parallel suggestion was made by Olson et al. (2006), who found impairment on a test of object-location memory in amnesic patients; it remains unclear, however, how generalizable such impairments might be for different types of relational information.

In two experiments reported here, memory for different kinds of relations (i.e., spatial and nonspatial relations) is tested with different paradigms under long- and short-lag conditions. On most accounts, performance should be impaired at longer lags, and intact at the shortest lag, but by emphasizing the need for relational memory might we also find impairment at the shortest possible lags? Such an outcome would conflict with claims that the role of the hippocampus in memory only emerges after many seconds have elapsed or many items have intervened, and would strengthen claims about the critical role of the hippocampus in relational memory.

\section{Materials and Methods \\ Participants}

Participants in both experiments were six patients (three male, three female) with amnesia, and six neurologically intact comparison partici- 


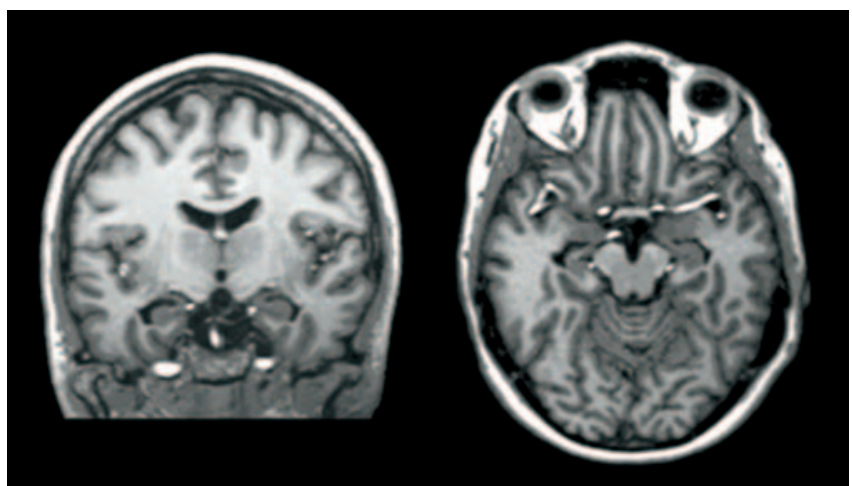

Figure 1. Magnetic resonance scans of hippocampal amnesic patient 0001. The images are coronal (left) and transverse (right) slices through the midportion of the hippocampus. They are from four sets of high-resolution T1-weighted (magnetization prepared rapid gradient echo) scans that have been averaged together to optimize gray-white contrast. Volume changes can be noted in the region of the hippocampus bilaterally.

pants, each matched to one of the patients individually with respect to age, education, and intelligence quotient (IQ). Five of the amnesic patients were drawn from the Patient Registry of the Division of Cognitive Neuroscience at the University of Iowa, and one patient (0001) was seen at the University of Illinois. Participants in the comparison group were recruited from the Champaign-Urbana community. All procedures were approved by the ethics committees at the University of Illinois and the University of Iowa, and informed consent was obtained from each participant.

For all patients, amnesia was secondary to an anoxic/hypoxic episode. In three patients, this was caused by cardiac or cardiopulmonary arrest; in the other three, the cause was an episode of seizures (leading in two cases to status epilepticus). Structural magnetic resonance imaging (MRI) examinations were completed on five of the patients, confirming bilateral hippocampal damage in each case. One of the patients (2563) wears a pacemaker and is consequently unable to undergo MRI examination; the anatomical analysis for this patient was based on a computerized tomography scan, and the only visible damage was in the hippocampal region. For patient 0001 (for whom we do not have volumetric scores), coronal and transverse sections from magnetic resonance imaging, through the mid-portion of the hippocampus, are shown in Figure 1. Hippocampal volume changes are evident, with bilateral hippocampal reduction clearly visible in the images.

In four patients, high-resolution volumetric MRI analyses were conducted for the entire brain (Allen et al., 2006). These analyses showed hippocampal volumes significantly decreased for each patient, with the studentized residual differences in hippocampal volume relative to a matched comparison group down by at least 2.6 and as much as 4.2 $z$-scores (Table 1). It was possible in these cases to conduct a thorough examination of areas of the brain both within and outside the MTL. These analyses showed that patient 2363 has some impairment in overall cerebrum gray matter, and in parietal gray matter; 1606 has some impairment in temporal lobe gray matter (where impairment refers to values more than two SDs below the mean of the comparison group), and is the only patient for whom we cannot confidently exclude damage to the perirhinal cortex. Although, critically, none of the four is more than two SDs below normal in frontal measures (Allen et al., 2006). Moreover, the impairment of the hippocampus in all four cases is disproportionate, even compared with other MTL regions (namely, the amygdala) and the remainder of the temporal lobe (gray and white matter).

All patients had memory impairments that were sufficiently severe to interfere with daily life, including preventing them from being employed since the onset of their amnesia. Each patient completed neuropsychological testing, which confirmed selective memory impairment disproportionate to deficits in general cognitive or intellectual functioning $(\mathrm{Ta}-$ ble 1). Their performance on the Wechsler memory scale-III (WMS-III) was at least 25 points lower than their performance on the Wechsler adult intelligence scale-III (mean full-scale IQ minus general memory index difference, 31.2), and the average delay score on the WMS-III (65.3) was more than two SDs below population means. They were also severely impaired in delayed recall on standardized tests such as the complex figure task. In contrast, and consistent with the absence of significant frontal-lobe impairment in the neuroanatomical data, performances on several standardized tests designed to assess working memory for items were mostly intact (Table 1). Patient 1606 had a working memory index score one SD below his full-scale IQ, but the score is an SD above his delayed recall index, and he performed normally on other working memory measures.

\section{Experiment 1: memory for the relations among items in scenes}

Past experiments from our lab have used eye-movement methodology to explore the status of relational memory representations that are to be maintained over the course of a very brief delay in work involving presentation of scenes that were exact matches or were manipulated versions of scenes viewed just seconds earlier (Ryan and Cohen, 2004a,b). Results showed that amnesic patients were like neurologically intact participants in directing more eye movements to the region of change in scenes that underwent a relational manipulation, but they were unlike the control participants in failing to exhibit all the changes in overall patterns of viewing normally elicited by manipulated scenes, despite the very short delays. Because behavioral measures were not obtained, it is unclear whether or not amnesic patients could explicitly identify the relational changes when manipulated scenes were presented. In experiment 1 , here, we use a direct (i.e., yes/no recognition) test of memory to explore the integrity of memory for relations among items embedded in scenes at both short and long lags.

Stimuli and design. The stimuli were 48 unique rendered scenes created using Punch! (Kansas City, MO) home design software, and sized to $800 \times 600$ pixels. Two versions of each scene, the original scene and a manipulated version of that scene involving a manipulation of the spatial relations among scene elements, were created, producing a total of 96 stimuli (Fig. 2, top). One item in each of the original scenes was designated a "critical item" and it underwent a spatial manipulation in the manipulated version of that scene, such that it occupied a different, albeit equally plausible, spatial location. Each critical item was presented in the context of just one scene, and across scenes the critical item moved equally often from left (in the original scene) to right (in the manipulated scene) as right to left.

There were two experimental blocks, each block consisting of 24 study trials (the original scenes) and 24 probe trials ( 12 scenes that were repeated versions of original scenes and 12 that were manipulated versions). A continuous recognition task was used in which probe trials were interleaved systematically among the study trials. Probe trials either appeared immediately after the corresponding study trial (lag 1), five trials later (lag 5), or nine trials later (lag 9).

On every trial, the scene was presented along with two questions, appearing sequentially at the bottom of the screen. One was an orienting question, different for each scene, which drew the viewer's attention to the "critical item" in that scene (e.g., for the first scene in Fig. 2, top: "Are the three lights on the ceiling directly above the chair?"). Half of the scenes were associated with orienting questions that elicited "yes" responses, and the remainder were associated with orienting questions that elicited "no" responses. The exact same orienting question was paired with corresponding study/probe scenes, and each question was created so that the same response would be given when the original scene was presented as when the manipulated version of that scene was presented. Consequently, verbal responses to the orienting question would not be informative to the participant as to whether or not the scene had undergone a manipulation. Then the memory question was presented, identical for each scene, asking whether or not the scene had been presented earlier in the experimental block, and if so, whether any of the items in the scene had changed locations.

Scenes were randomly assigned to lists, and counterbalancing was con- 
Table 1. Patient characteristics, hippocampal volume reductions, and neuropsychological test scores

\begin{tabular}{|c|c|c|c|c|c|c|c|c|c|c|c|c|}
\hline \multirow[b]{2}{*}{ Subject } & \multirow[b]{2}{*}{ Handedness } & \multirow{2}{*}{$\frac{\text { Hippocampus }}{\text { z-score }}$} & \multicolumn{3}{|c|}{ WAIS-III } & \multicolumn{3}{|c|}{ WMS-III } & \multicolumn{4}{|c|}{ Working memory } \\
\hline & & & VIQ & PIQ & FSIQ & GMl & DRI & CFT & WMI & Digit Span & Arith & Sent Rept \\
\hline 0001 & $\mathrm{R}$ & $\mathrm{N} / \mathrm{A}$ & 83 & 100 & 90 & 54 & 64 & 2 & 93 & 8 & 6 & $\mathrm{~N} / \mathrm{A}$ \\
\hline 1606 & $\mathrm{R}$ & -3.99 & 94 & 89 & 91 & 66 & 61 & 11 & 76 & 7 & 9 & 8 \\
\hline 1846 & $\mathrm{R}$ & -4.23 & 89 & 79 & 84 & 57 & 62 & 6 & 85 & 10 & 7 & 11 \\
\hline 2144 & $\mathrm{R}$ & -3.92 & 102 & 94 & 99 & 56 & 57 & 3 & 99 & 11 & 9 & 12 \\
\hline 2363 & $\mathrm{R}$ & -2.64 & 112 & 83 & 98 & 73 & 74 & 5 & 88 & 8 & 11 & 10 \\
\hline 2563 & $\mathrm{~L}$ & $\mathrm{~N} / \mathrm{A}$ & 91 & 98 & 94 & 63 & 74 & 7 & 99 & 14 & 6 & 13 \\
\hline
\end{tabular}

Hippocampal z-scores represent the combined (left and right hemisphere) studentized residuals of hippocampal volume relative to a group of 43 comparison subjects (Allen et al, 2006 ). WAIS-III, Wechsler adult intelligence scale-III; VIQ, verbal IQ; PIQ, performance IQ; FSIQ, full-scale IQ; WMS-III, Wechsler memory scale-III; GMI, general memory index; DRI, delayed recall index. For the WMS-III, the DRI is an average of the auditory delayed index and visual delayed index. These tests yield mean scores in the normal population of 100 with an SD of 15. CFT, Complex figure test [delayed recall raw score (total possible correct = 36)]; WMI, working memory index; WMS-III, Wechsler memory scale-III; DS, digit span subtest; WAIS-III, age-corrected scaled score; Arith, arithmetic subtest; WAIS-III, age-corrected scaled score; Sent Rept, sentence repetition subtest, multilingual aphasia examination, raw score.

ducted such that each list rotated across experimental conditions (repeated vs. manipulated), lags $(1,5$, and 9), and experimental blocks (block 1 and block 2). For manipulated scenes, the critical item moved from left to right and from right to left equally often at each lag and within each experimental block for every participant.

Procedure. After obtaining informed consent and providing detailed instructions, each participant was tested individually. Participants were asked to provide oral responses to both the orienting question, inquiring about visible relations among critical objects, and the memory question, inquiring about whether or not the scene had been presented previously and about whether or not it had been manipulated, sequentially on each trial. The instructions were repeated between experimental blocks.

Each trial began with the presentation of a centrally located cross-hair, which remained on the screen for $3 \mathrm{~s}$, serving as a critical buffer between displays so as to ensure that participants would not "see" the critical item move from one location to another when the original scene was followed by a manipulated version of that scene in the absence of intervening trials (i.e., lag 1 trials). Then the scene was presented for $20 \mathrm{~s}$. Over the course of each $20 \mathrm{~s}$ trial, the scene was presented alone for $5 \mathrm{~s}$, the orienting question was then presented along with the scene for $6 \mathrm{~s}$, and finally, the orienting question was replaced with the memory question, which remained on the screen along with the picture for the remaining $9 \mathrm{~s}$. In addition to the questions appearing visually on the screen, the experimenter read the questions aloud. If the response was not provided while the corresponding question was still visible, the participant was prompted by the experimenter. Participants were allowed to take breaks, as necessary, in between the experimental blocks.

All participants completed this experiment on two separate occasions, separated in time by several months. Each session consisted of a different counterbalanced order of the experiment. Debriefing was provided at the end of the final session.

Statistical analyses. Data for the test of relational memory are reported only for those trials associated with a correct response to the orienting question (anoxic patients, 97.3\% correct; comparison group, $99.0 \%$ correct) and correct identification of a previously viewed scene as old (anoxic patients, 93.6\% correct; comparison group, $99.5 \%$ correct). ${ }^{a}$

The proportion of correct relational manipulation judgments was calculated for each participant at each lag using the following formula: (hits + correct rejections)/2, where hits refers to correct identification of manipulated scenes, and correct rejections refers to correct identification of unchanged (repeated) scenes.

${ }^{a}$ Patient 1606 , for whom we could not rule out the possibility of perirhinal cortex damage, responded correctly to the orienting question on $97.4 \%$ of the trials, a level of performance equivalent to the mean for the remaining patients (97.3\% correct; SD, 1.45). Although, of interest, patient 1606 performed more poorly than every other patient on the test of scene memory (i.e., identifying a previously viewed scene as old; patient 1606, $83.9 \%$ correct; amnesic group mean, $95.4 \%$ correct; SD, 4.39) 
Because of possible violations of the homogeneity of variance associated with analysis of binary data summarized as proportions or percentages, an arcsine transformation was performed and statistical tests were conducted using the transformed data. Further, to improve the equality of variance, a correction was applied if performance was perfect (i.e., proportion correct, 1.0) before applying the arcsine transformation. The formula used to correct perfect scores was as follows: $(n-1 / 4) / n$, where $n=$ total number of trials.

\section{Experiment 2: memory for face-scene relations}

Experiment 2 extends the current work beyond the spatial domain, examining memory for the relations among arbitrarily paired faces and scenes, tested under both short- and long-lag conditions. In addition, other methodological differences between this paradigm and the one used in the previous experiment will further test the generality of the current findings.

Stimuli and design. The stimuli used in experiment 2 were 36 male and 36 female full-color face images selected from our faces database (Althoff and Cohen, 1999), and 72 full-color images of real-world scenes from a commercial image collections company, Brand X Pictures (Madison, WI). Each face was sized to $280 \times 280$ pixels and placed on a uniform gray background that measured $300 \times 300$ pixels; the scenes measured $800 \times 600$ pixels. These stimuli were used to generate the study displays, each consisting of a face superimposed on a scene to create a trial-unique face-scene pair, and the probe displays, each consisting of three previously viewed faces superimposed on a previously viewed scene (Fig. 2, bottom).

Participants received two experimental blocks, each consisting of 36 study trials and 12 probe trials. Memory for the studied face-scene pairs was assessed using probe trials, in which three previously viewed faces were combined with a previously viewed scene, and participants were asked to identify the one face that had been studied with (and thus matched) that scene. The faces in each test display had been presented previously an equal number of times. Thus, item memory (memory for individual faces) would not be sufficient to guide successful performance; instead, correct responding would require memory for facescene pairings (i.e., require relational memory).

The probe trials were interleaved systematically among the study trials, appearing either immediately after the corresponding study trial (lag 1) or nine study trials later (lag 9). Half of the probe trials were match displays in which one of the three faces had been studied with that scene. The other half were re-pair displays in which, although all three faces had been studied previously, none had been studied with that scene. Data from the re-pair displays are not presented here because those displays were included in the experiment only for a control condition for eye movement measures that are not reported in this study.

Faces and scenes were randomly assigned to lists, and counterbalancing was conducted such that each list of faces was seen equally often with each list of scenes; these lists rotated across conditions, so as to be seen equally often for the different probe-display types (match and re-pair), lags (1 and 9), and experimental blocks (block 1 and block 2). Female and male faces were segregated and presented in separate experimental blocks; across participants they were seen equally often in the first or second block. The matching face in match displays appeared equally often in each spatial position (left, right, and bottom).

Procedure. After obtaining informed consent, providing detailed instructions, and conducting as many practice trials as necessary to be sure that each participant consistently identified the intended face in a threeface display with the appropriate key press response, each participant was tested individually. Participants were asked to commit each face-scene pair to memory in anticipation of the presentation of occasional threeface probe displays. They were instructed to respond to each three-face display by pressing a button corresponding to the position occupied by the face that had been paired with (i.e., matched) that scene. It was emphasized that they should select a face on every trial, even if they did not believe that any of the faces had been paired with that scene previously (e.g., when re-pair displays were presented). This requirement ensured that participants would provide a response on every trial, even when they could not identify the matching face with certainty. A written reminder was affixed to the bottom of the computer screen to remind the patients that they were to "identify the matching face" when each threeface display was presented; this reminder indicated the correspondence between the spatial location of a face and the key with which a response was to be made. These instructions were repeated between experimental blocks.

Each trial began contingent on the participant fixating a centrally located cross-hair. On each study trial, a scene was presented alone for $3 \mathrm{~s}$, then a single face was superimposed on the center of the scene, and the pair remained on the screen for an additional $5 \mathrm{~s}$. On each probe trial, a scene was presented alone for $3 \mathrm{~s}$ and then a three-face display was superimposed on it; the faces and scene remained on the screen for $10 \mathrm{~s}$, and responses were to be made during this time. Participants could take a break between blocks as necessary.

Patients were each tested on five separate occasions, with different combinations and orders of the stimulus materials to procure stable, reliable estimates of their performance abilities. Each session was separated by at least 1 month. However, because of concerns that the memory abilities of the comparison participants might be good enough that multiple exposures to the same stimuli might influence their performance in a way that would be unlikely in the memory-impaired amnesic patients, no comparison participant was tested more than two times. Debriefing was provided at the end of the final session.

Statistical analyses. The proportion of correct responses (i.e., correct identification of the matching face) was calculated for each participant at each lag. As in experiment 1, an arcsine transformation was performed and statistical tests were conducted using the transformed data. Again, to improve the equality of variance, a correction was applied if performance was perfect (i.e., proportion correct, 1.0) before applying the arcsine transformation.

\section{Results}

\section{Experiment 1: memory for the relations among items in scenes}

\section{Relational memory}

Relative to the comparison group, amnesic patients were impaired on memory for spatial relations $\left(F_{(1,10)}=59.68\right.$; $p<$ 0.001). Consistent with previous findings (Holdstock et al., 1995, 1999, 2000a,b; Buffalo et al., 1998), the memory deficit was lagsensitive (i.e., greater at longer lags; interaction of lag $\mathrm{x}$ group, $F_{(2,20)}=6.24, p=0.008$ ) (Fig. 3, top). Amnesic patient performance dropped precipitously across lags, whereas performance for comparison subjects dropped little (Table 2). Planned comparisons revealed that amnesics were impaired at lags 5 and 9 $\left(t_{(10)}=6.74, p<0.001\right.$ and $t_{(10)}=7.70, p<0.001$, respectively), with performance at these longer lags not significantly greater than chance $\left(t_{(5)}=0.026, p=0.98\right.$ and $t_{(5)}=0.70, p=0.52$, respectively). These results indicate that this task successfully tapped into the type of memory for which the hippocampus is critical, namely relational memory, and that it revealed the typical signature of long-term memory deficit. These circumstances provide an ideal opportunity to evaluate short-lag performance.

Critically, amnesics were also impaired even at lag 1. Despite the absence in this condition of any intervening items between study and test trials, planned comparisons showed that amnesics were impaired on memory for spatial relations $\left(t_{(10)}=3.84 ; p=\right.$ 0.005) (Fig. 3, top). ${ }^{b}$

${ }^{b}$ Because the measure of performance that was used to explore memory for relations, [(hits + correct rejections)/2], assumes that memory is a high-threshold process, statistics were also performed on $d^{\prime}$ scores. Consistent with the results reported above, amnesic patients were impaired on the test of spatial relational memory $\left(F_{(1,10)}=77.34\right.$; $p<0.001$ ), and this impairment was lag sensitive (lag by group interaction: $\left.F_{(2,20)}=9.84 ; p=0.001\right)$. Similarly, planned comparisons indicate that this impairment is evident at every $\operatorname{lag}\left(\operatorname{lag} 1, t_{(10)}=3.84, p=0.003\right.$; $\operatorname{lag} 5$, $\left.t_{(10)}=7.82, p<0.001 ; \operatorname{lag} 9, t_{(10)}=8.09, p<0.001\right)$. 


\section{EXPERIMENT 1}

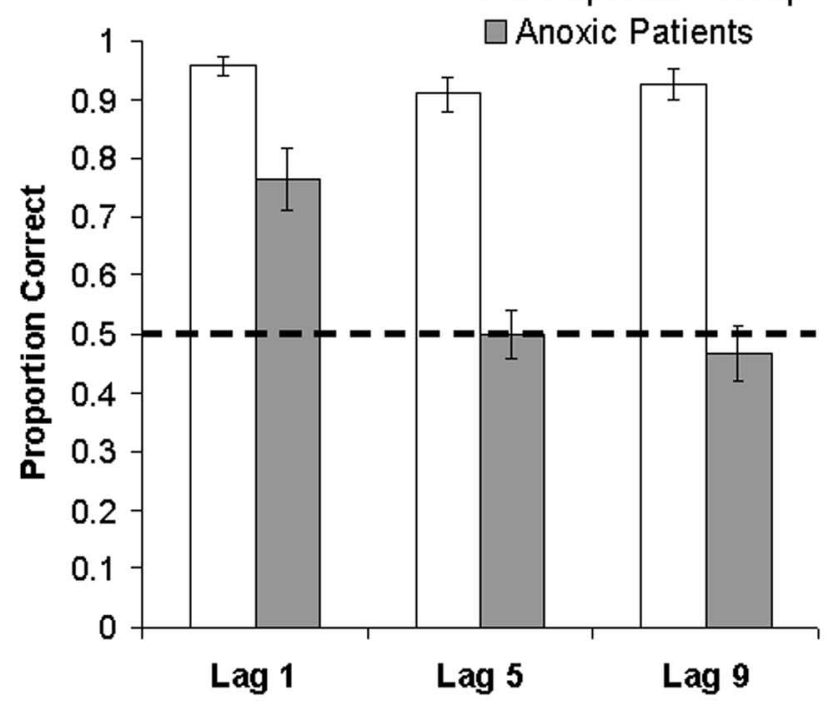

\section{EXPERIMENT 2}

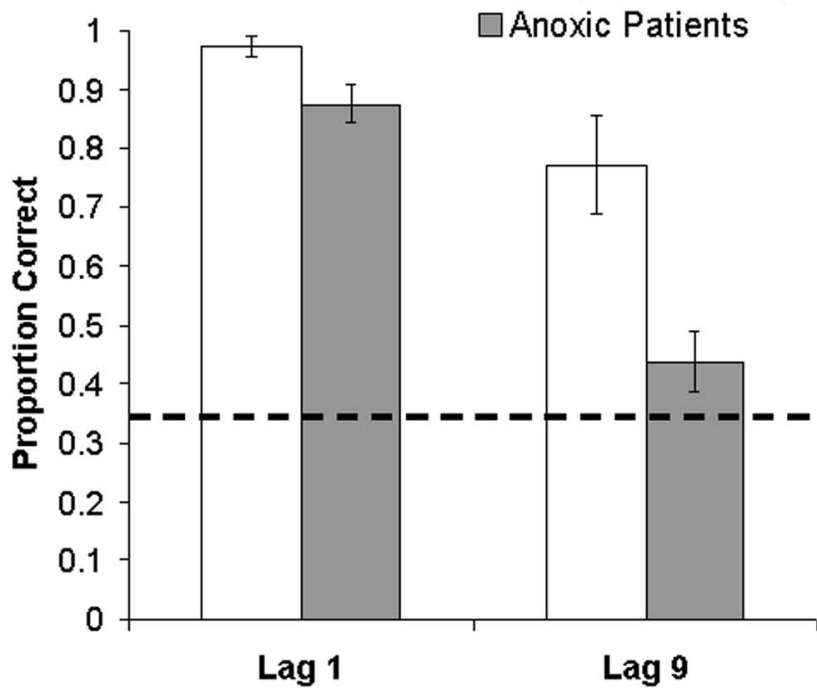

Figure 3. Deficit in relational memory in amnesia. The proportion of correct relational memory judgments for the comparison group (white bars) and the amnesic patients (gray bars) in experiment 1 (top) and experiment 2 (bottom) are shown. SE bars are plotted around the means. The dashed line represents chance performance.

Table 2. Performances on tests of item (scene) and relational memory for experiment 1

\begin{tabular}{lllll}
\hline & Lag 1 hits & Lag 5 hits & Lag 9 hits & 0verall CR \\
\hline $\begin{array}{c}\text { Scene memory } \\
\text { Comparison group }\end{array}$ & $0.995(0.013)$ & $0.995(0.013)$ & $0.995(0.013)$ & $0.984(0.012)$ \\
$\quad \begin{array}{l}\text { Amnesic patients } \\
\text { Relational memory }\end{array}$ & $0.990(0.026)$ & $0.937(0.084)$ & $0.883(0.111)$ & $0.697(0.145)$ \\
$\quad$ & & & & \\
Comparison group & $0.989(0.027)$ & $0.906(0.102)$ & $0.924(0.076)$ & $0.922(0.067)$ \\
Amnesic patients & $0.701(0.276)$ & $0.402(0.264)$ & $0.366(0.312)$ & $0.670(0.136)$
\end{tabular}

Mean performance on the tests of item and relational memory for the comparison group and the amnesic patients. Hit rates, broken down as a function of lag, are presented along with the overall correct rejection (CR) rate for each condition. SDs are shown in parentheses.

Notably, patient 1606, for whom we could not confidently exclude the possibility of perirhinal cortex damage, performed well above the group mean on the test of relational memory at lags 1 (1606, 92.9\% correct; amnesic patients, 73.1\% correct; SD,
11.8 ) and 9 (1606, 63.0\% correct; amnesic patients, $43.4 \%$ correct; SD, 9.3), although slightly below the group mean at lag 5 (1606, 42.6\% correct; amnesic patients, $51.3 \%$ correct; SD, 10.5).

Relational versus scene memory

To determine whether the impairments observed in experiment 1 were specifically relational in nature, and not attributable to a more global memory deficit, we contrasted memory for the relations among items embedded in scenes to memory for the scenes themselves. Because novel scenes (i.e., scenes being presented for the first time in the experimental block) cannot be meaningfully classified as a function of lag, performances on the tests of relational and scene memory were collapsed across lag for the purposes of this analysis; the same measure of performance described previously was also used here [i.e., (Hits $+\mathrm{CR}$ )/2 with an arcsine transformation applied to the data]. A two by two ANOVA with the factors group (amnesic patients and comparison participants) and condition (relations and scenes) was performed. Amnesic patients performed more poorly than the matched comparison group (main effect of group: $F_{(1,10)}=71.66 ; p<0.001$ ) and both groups performed worse on the relational memory test than the test of scene memory (main effect of condition: $F_{(1,10)}=$ $37.20 ; p<0.001)$. Critically, there was also a marginal group by condition interaction $\left(F_{(1,10)}=3.41 ; p=0.09\right)$ suggesting that any impairment on the test of relational memory was disproportionate to that on the test of memory for scenes (Table 2). To test this possibility directly, performances of each patient on the tests of relational and scene memory were subtracted from the performances of their individually matched comparison participants, and the arcsine transformed difference scores for these tests of memory were compared. This analysis confirmed that performance on the test of relational memory was compromised to a greater extent than performance on the test of memory for scenes $\left(t_{(5)}=4.57 ; p=0.006\right)$ suggesting that these patients have a disproportionate deficit on tests of memory that depend on information about relations among items embedded in complex scenes. $^{c}$

\section{Experiment 2: memory for face-scene relations}

The results of experiment 1 indicate that the hippocampus is critically involved in memory for spatial relations among scene elements, even when study and probe trials are presented consecutively and only seconds apart. But is this deficit a consequence of a general relational memory requirement or is it specific to spatial relations? Although our relational memory account of hippocampal function has emphasized generality across materials and modalities (Cohen and Eichenbaum, 1993; Eichenbaum and Cohen, 2001), some influential models of hippocampal function suggest that the hippocampus is particularly important for spatial relations (O'Keefe and Nadel, 1978; Kumaran and Maquire, 2005), and the results of experiment 1 cannot adjudicate between these views. Strong evidence for a general role of the hippocampus in relational memory at both long and short lags will be evident to the extent that we are able to replicate the results of the previous experiment using nonspatial materials, namely arbitrarily paired faces and scenes. Relative to the comparison group, amnesic patients were impaired on memory for face-scene relations $\left(F_{(1,10)}=13.53 ; p=0.004\right)$. As in experiment 1 , this deficit was lag-sensitive (i.e., greater at longer lags; interaction of lag by group, $F_{(1,10)}=4.87, p=0.05$ ) (Fig. 3, bottom). Amnesic perfor-

The level of performance on the test of item memory among amnesic patients may not be fully apparent in the available data because the comparison group performed at ceiling when memory for the scenes was tested. 
mance dropped from 0.874 to 0.438 from lag 1 to lag 9 , compared with performances of 0.972 and 0.771 , respectively, for the comparison participants. Again, planned comparisons revealed that amnesics were impaired under longer lag conditions (lag 9: $t_{(10)}$ $=3.40 ; p=0.007)$ with performance not significantly greater than chance $\left(t_{(5)}=2.03 ; p=0.098\right)$.

Having thus found the typical signature of long-term memory deficit, here for nonspatial (face-scene) relations, we can turn to the question of performance at the shortest lag. Consistent with the results of experiment 1, hippocampal amnesics were also impaired, even at lag 1. Planned comparisons showed that despite the absence of any intervening items between study and test trials, amnesics were impaired on memory for face-scene relations $\left(t_{(10)}=2.72 ; p=0.02\right)$ (Fig. 3, bottom).

Patient 1606 performed comparably to the patients with more selective hippocampal damage at both lags (lag 1: 1606, 85.7\% correct; amnesic group, 87.7\% correct; SD, 7.8; lag 9: 1606, 44\% correct; amnesic group, $43.7 \%$ correct; SD, 12.8).

\section{Discussion}

General consensus in neuropsychology has been that the hippocampus is not required for maintenance of information for brief periods of time and/or in the absence of distraction (Cave and Squire, 1992; but see Holdstock et al., 1995; Buffalo et al., 1998). However, past investigations of very short-delay or shortlag memory may not have adequately targeted aspects of memory that are critically hippocampal-dependent (i.e., memory for relations among items).

Some very recent work on memory for spatial relations has raised questions about the standard view. In an eye movement experiment in which memory for relations was tested at very short delays using scenes that were repeated versus scenes that underwent relational manipulations, patients with hippocampal amnesia showed the same tendency as neurologically intact viewers to direct disproportionately more viewing to the region of change of manipulated scenes (as compared with viewing of the same region when the scene was unchanged), despite the same patients failing to show this relational manipulation effect at longer delays (Ryan et al., 2000; Ryan and Cohen, 2004a). Yet, in contrast to this finding of seemingly intact short-delay relational memory in amnesia, the patients failed to show all the eye movement differences for manipulated versus repeated scenes exhibited by neurologically intact participants, specifically on measures of overall viewing, in the short-delay task (Ryan and Cohen, 2004b). Without obtaining concomitant behavioral measures, it could not be determined whether or not the patients could explicitly identify the changes that had taken place.

Behavioral performance at short-delays has begun to be examined in hippocampal amnesia. In one recent experiment, a selective impairment in memory for object-location associations was observed at very short delays in hippocampal amnesic patients (Olson et al., 2006). Memory for object-location relations was also tested in an experiment in which participants viewed an array of objects for a study period and were subsequently asked to place the objects in the appropriate spatial locations (Crane and Milner, 2005). Lobectomy patients with extensive rightlateralized MTL lesions, including hippocampal involvement, were impaired even when the placement test was administered just after the associated study exposure. However, previous work by the same laboratory conducted with a similar group of patients found intact short-delay memory performance for object-location relations when participants were shown manipulated versions of studied scenes and were asked to identify the changes (Pigott and Milner, 1993). Accordingly, the status of short-lag, short-delay memory for relations has remained ambiguous.

Findings from the experiments reported here document clearly that the hippocampus is critically involved in supporting performance not only at longer lags (where performance fell to chance levels), but even in the shortest-lag, shortest-delay (lag-1) condition. Moreover, they show that this role generalizes across different kinds of relations (spatial and nonspatial) and testing paradigms.

These findings clearly seem at odds with the historical view of amnesia as being a selective deficit in long-term memory. However, although contrasting with the emphasis from neuropsychology on selective hippocampal involvement in long-term memory, the current findings complement recent fMRI findings of hippocampal recruitment in tasks typically thought to be the province of working memory (Ranganath and D'Esposito, 2001; Cabeza et al., 2002; Park et al., 2003; Karlsgodt et al., 2005; but see Zarahn et al., 2005).

Possible explanations of the current findings, along with an account of related neuroimaging findings, may shed light on important issues concerning the role of the hippocampus in memory, the nature of the deficit in amnesia, and the distinction between long-term and short-term (or working) memory. One possible way to understand the impairment seen here at even the shortest lag is that the patients failed to process the scenes and, more particularly, the relations among items in the scenes sufficiently well at the time of initial study to enable subsequent identification of a relational change at any lag. Recent work suggests that under some circumstances the perirhinal cortex, and even the hippocampus itself, may be involved in high-level perceptual processing and/or representation of feature conjunctions or complex scenes (Bussey et al. 2002; Lee et al., 2005a,b; for review, see Buckley and Gaffan, 2006), and the materials used here were sufficiently complex, especially the scenes in experiment 1 , to place considerable challenge on such perceptual/representational abilities.

Although we cannot completely rule out the possibility that some impairment in perceptual representation abilities contributes to deficits in performance on tests of relational memory used here, the inclusion in experiment 1 of orienting questions and of a test of memory for the scenes themselves provide some leverage against the perceptual representation explanation of the current findings. ${ }^{d}$ All of the patients consistently provided correct responses to the orienting questions that tested on-line perception of the very relations tested subsequently in the relationalmemory (match/mismatch) task. This was true even for patient 1606 , for whom we cannot rule out the possibility of perirhinal cortex damage. Also, the patients showed a disproportionate impairment on the test of relational (vs scene) memory in experiment 1 , although it bears mentioning that comparison participants performed at ceiling on the test of scene memory, which may have resulted in the underestimation of any item memory impairment. Nonetheless, patients proved fully capable of identifying previously viewed scenes as old. ${ }^{e}$ In fact, the relational memory data reported here were only for those trials on which

${ }^{d}$ This datum alone might not be the best indicator of intact perceptual processing; neurologically intact comparison participants may use different (more detailed) information to answer these questions because they process the scenes in a qualitatively different manner. Because this is a potential concern, future work should incorporate other, more sensitive measures to address this issue.

${ }^{e}$ However, patient 1606 showed the poorest performance on the test of scene memory of all the amnesic patients, which is likely attributable to perirhinal cortex damage in this individual. 
participants answered both the orienting question and the scene memory (old/new) question correctly. Accordingly, it does not seem that the short-lag relational memory impairments observed here could be caused by the failure to actually process the information in the scenes and form perceptual representations of them. Finally, although Lee et al. (2005a,b) have reported impairments on tests of scene perception in patients with bilateral damage restricted to the hippocampus, the possibility that some memory requirement (i.e., maintaining information across subsequent fixations) is responsible for the observed impairment cannot be ruled out. More research on this issue will help to sort out the relationship between perceptual representations, their retention over time, and the role played in these processes by hippocampus and other MTL structures.

A second, and seemingly more direct, way to interpret the impairment seen here at even the shortest lag is as providing evidence that the relational memory deficit in amnesia previously demonstrated for long-term memory extends to short-term or working memory as well [i.e., the hippocampus plays a critical role in supporting both long-term and short-term (or working) relational memory]. This view attributes performance on the long-lag trials here to long-term relational memory and performance on the short-lag trials here to short-term relational memory, both supported by the hippocampus. If this is correct, it calls into question the long-term/short-term memory distinction. Historically, the apparent selectivity of the deficit in amnesia to long-term memory, leaving short-term memory intact, has been central to support for the long-term/short-term memory distinction. Findings like the one here that long-lag (or long-term memory) deficits and short-lag (or short-term memory) deficits associate rather than dissociate, at least for relational memory, undermines the support that findings from amnesia can offer to distinction claims. When considered together with the recent neuroimaging findings of hippocampal recruitment not only for long-term memory tasks but also for working memory tasks, again speaking to association rather than dissociation, additional tension is placed on claims of distinction between long(er)-term and short(er)-term memory systems (Ranganath and Blumenfeld, 2005).

Finally, a third possible account of memory impairment seen here at even the shortest lag is that it reflects the failure of amnesic patients to make use of the normal contributions of long-term memory to performance regardless of lag or delay. That is, even if for simple materials, short-lag short-delay performance may be supported fully by short-term or working memory mechanisms, the contributions of a larger capacity long-term memory system (and of the hippocampus) may be manifested at even the shortest lags and delays if the materials are sufficiently complex. Indeed, Ryan and Cohen (2004b) have emphasized that working memory and long-term memory each make contributions to the on-line representation of scenes, such as the ones used in the current study. On this view, it is important to avoid assuming that shortlag and long-lag performances can be attributed directly and exclusively to short-term and long-term memory mechanisms, respectively. This may be particularly true when, as in the current work, there is interleaving of different lag trials, which could encourage the use of long-term memory strategies throughout and dissuade participants from attempting to actively maintain information over the delay, rather than separating lags into different experimental blocks.

Taken together, it is evident that, at least when relational memory is required, the hippocampus plays a critical role in performance not only under long-delay and long-lag conditions but even at short delays and in the absence of any intervening items between study and test. These findings suggest that characterizing the memory functions of the hippocampus may have less to do with any distinction between long-term and short-term (or working) memory than it has to do with the distinction between relational memory and memory for items.

\section{References}

Allen J S, Tranel D, Bruss J, Damasio H (2006) Correlations between regional brain volumes and memory performance in anoxia. J Clin Exp Neuropsychol 28:457-476.

Althoff RR, Cohen NJ (1999) Eye-movement-based memory effect: a reprocessing effect in face perception. J Exp Psychol Learn Mem Cogn 25:1-14.

Baddeley AP, Warrington EK (1970) Amnesia and the distinction between long and short-term memory. J Verb Learn Verb Behav 9:176-189.

Buckley MJ, Gaffan D (2006) Perirhinal cortical contributions to object perception. Trends Cogn Sci 10:100-107.

Buffalo EA, Reber PJ, Squire LR (1998) The human perirhinal cortex and recognition memory. Hippocampus 8:330-339.

Bussey TJ, Saksida LM, Murray EA (2002) Perirhinal cortex resolves feature ambiguity in complex visual discriminations. Eur J Neurosci 15:365-374.

Cabeza R, Dolcos F, Graham R, Nyberg L (2002) Similarities and differences in the neural correlates of episodic memory retrieval and working memory. NeuroImage 16:317-330.

Cave C, Squire LR (1992) Intact verbal and non-verbal short-term memory following damage to the human hippocampus. Hippocampus 2:151-163.

Cohen NJ (1984) Preserved learning capacity in amnesia: evidence for multiple memory systems. In: The neuropsychology of memory (Butters N, Squire LR, eds), pp 83-103. New York: Guilford.

Cohen NJ, Eichenbaum H (1993) Memory, amnesia, and the hippocampal system. Cambridge, MA: MIT.

Cohen NJ, Squire LR (1980) Preserved learning and retention of patternanalyzing skill in amnesia: dissociation of knowing how and knowing that. Science 210:207-210.

Crane J, Milner B (2005) What went where? Impaired object-location learning in patients with right hippocampal lesions. Hippocampus 15:216-231.

Eichenbaum H, Cohen NJ (2001) From conditioning to conscious recollection: memory systems of the brain. Oxford: Oxford UP.

Holdstock JS, Shaw C, Aggleton JP (1995) The performance of amnesic subjects on tests of delayed matching-to-sample and delayed matching-toposition. Neuropsychologia 33:1583-1596.

Holdstock JS, Mayes AR, Cezayirli E, Aggleton JP, Roberts N (1999) A comparison of egocentric and allocentric spatial memory in medial temporal lobe and korsakoff amnesics. Cortex 35:479-501.

Holdstock JS, Mayes AR, Cezayirli E, Isaac CL, Aggleton JP, Roberts N (2000a) A comparison of egocentric and allocentric spatial memory in a patient with selective hippocampal damage. Neuropsychologia 38:410-425.

Holdstock JS, Gutnikov SA, Gaffan D, Mayes AR (2000b) Perceptual and mnemonic matching-to-sample in humans: contributions of the hippocampus, perirhinal and other medial temporal lobe cortices. Cortex 36:301-322.

Karlsgodt KH, Shirinyan D, van Erp TG, Cohen MS, Cannon TD (2005) Hippocampal activations during encoding and retrieval in a verbal working memory paradigm. NeuroImage 25:1224-1231.

Kumaran D, Maguire EA (2005) The human hippocampus: cognitive maps or relational memory? J Neurosci 25:7254-7259.

Lee AC, Bussey TJ, Murray EA, Saksida LM, Epstein RA, Kapur N, Hodges JR, Graham KS (2005a) Perceptual deficits in amnesia: challenging the medial temporal lobe mnemonic view. Neuropsychologia 43:1-11.

Lee AC, Buckley MJ, Pegman SJ, Spiers H, Scahill VL, Gaffan D, Bussey TJ, Davies RR, Kapur N, Hodges JR, Graham KS (2005b) Specialization in the medial temporal lobe for processing of objects and scenes. Hippocampus 15:782-797.

O'Keefe J, Nadel L (1978) The hippocampus as a cognitive map. Oxford: Oxford UP.

Olson IR, Page K, Sledge Moore K, Chatterjee A, Verfaellie M (2006) Work- 
ing memory for conjunctions relies on the medial temporal lobe. J Neurosci 26:4596-4601.

Park DC, Welsh RC, Marshuetz C, Gutchess AH, Mikels J, Polk TA, Noll DC, Taylor SF (2003) Working memory for complex scenes: age differences in frontal and hippocampal activations. J Cogn Neurosci 15:1122-1134

Pigott S, Milner B (1993) Memory for different aspects of complex visual scenes after unilateral temporal- or frontal-lobe resection. Neuropsychologia 31:1-15.

Ranganath C, Blumenfeld RS (2005) Doubts about double dissociations between short- and long-term memory. Trends Cogn Sci 9:374-380.

Ranganath C, D'Esposito M (2001) Medial temporal lobe activity associated with active maintenance of novel information. Neuron 31:865-873.

Ryan JD, Cohen NJ (2004a) Processing and short-term retention of relational information in amnesia. Neuropsychologia 42:497-511.
Ryan JD, Cohen NJ (2004b) The nature of change detection and online representations of scenes. J Exp Psychol Hum Percept Perform 30:988-1015

Ryan JD, Althoff RR, Whitlow S, Cohen NJ (2000) Amnesia is a deficit in relational memory. Psychol Sci 11:454-461.

Scoville WB, Milner B (1957) Loss of recent memory after bilateral hippocampal lesions. J Neurol Neurosurg Psychiatry 20:11-12.

Warrington EK, Baddeley AD (1974) Amnesia and memory for visual location. Neuropsychologia 12:257-263.

Wickelgren WA (1968) Sparing of short-term memory in an amnesic patient: implications for strength theory of memory. Neuropsychologia 6:235-244

Zarahn E, Rakitin B, Abela D, Flynn J, Stern Y (2005) Positive evidence against human hippocampal involvement in working memory maintenance of familiar stimuli. Cereb Cortex 15:303-316. 